\title{
Channel estimation in space time block coded MIMO-OFDM system: a review
}

\author{
Rishi Choubey ${ }^{1 *}$ and V.B. Reddy ${ }^{2}$ \\ Research Scholar, Department of Electronics and Communication, Swami Vivekanand University, Sagar, Madhya \\ Pradesh, India ${ }^{1}$ \\ Associate Professor, Department of Electrical and Electronics, Swami Vivekanand University, Sagar, Madhya \\ Pradesh, India ${ }^{2}$
}

\begin{abstract}
This paper explores the opportunity in the direction of channel estimation in space time block coded orthogonal frequency-division multiplexing (OFDM) and multiple inputs and multiple outputs (MIMO) system. In this paper several aspects have been analysed and considered for the study. The main parameters analysed are the modulation scheme, channel capacity, channel variations and bit error rate (BER) impact. This study explores various aspects in the previous literature including the method, results and gaps. This study provides a way to explore the opportunity to extend the research work in the direction of improving the performance based on the parameter discussed.
\end{abstract}

\section{Keywords}

OFDM, MIMO, BER, Channel capacity.

\section{Introduction}

Orthogonal frequency-division multiplexing (OFDM) provides a single data stream modulation which can split across several channels with different frequencies. OFDM is a frequency division multiplexing (FDM) system which is beneficial in multi-channel communication [1]. FDM system is helpful in the division of bandwidth that is available for the spectrum [2]. The main work of this system is to combines a large number of low data rate carriers to construct a composite communication system of high data rate [3]. It provides a spaced closely without inter carrier interference (ICI) [4].

In today's circumstance multiple inputs and multiple outputs (MIMO) is to a great degree obliging with the mix of OFDM structure. The versatility of MIMO systems remembering the last objective to have high data rates is an especially captivating examination subject for future booking course of action sorts out and their applications. MIMO systems offer substantially more essential channel restrict over standard single-data single-yield structure. As of various transmit Algorithms have been passed on to experience past what many would consider conceivable in the MIMO systems [5]. MIMO system containing multiple antennas both at transmitter and receiver end.

*Author for correspondence

17
It has the capability to provide higher capacity in wireless communications [6,7]. Gathered qualities strategies, for event, space-time coding have gotten an enormous measure of thought in context of their ability to give higher efficiency [8, 9]. While applying this method in a repeat specific channel, a space-time equalizer is required at the receiver to change for the frequency and carrier signal [10].

This multipath spread reasons optional time diffusing, settling, and stage change, known as blurring, in the got flag [11-13]. Code division multiple access (CDMA) structure has the upsides of extending past what many would consider conceivable adjoining the resistance against staying [14-16]. In multi-customer CDMA structures, different multiple access interference (MAI) is viewed as one of the standard wellsprings of execution debasement. Versatile isolating frameworks have been sufficient used to level the direct and accordingly lessen the MAI in the direct sequence (DS)-CDMA structure [17-19].

\section{Related work}

In 2006, Hui et al. [20] indicated the problem of cross layer design in space-time block code (STBC)MIMO-OFDM wireless channels. They have evaluated the impact and performance of cross layer optimization. They have presented the mathematical 
expression and simulation result of cross layer resource allocation and scheduling for multi-user space-time coded MIMO-OFDM systems.

In 2007, Liu et al. [21] presented a model of spacefrequency block coded (SFBC) MIMO-OFDM system. They have analyzed channel estimation errors and BER.

In 2011, Yu et al. [22] suggested to take the advantages of the combination of MIMO, cognitive radio and OFDM systems. They have suggested that the orthogonal space-time block codes in MIMO has the capability of providing multiple transmit/receive antennas full diversity gain, minimizing the impact of multipath fading and gaining better BER performance, increasing the digital transmission rate at the same time. Their results also support the combination as it improves the signal to noise ratio (SNR).

In 2011, Gupta et al. [23] suggested the combination of MIMO and OFDM systems with wideband transmission to mitigate intersymbol interference for the performance improvement. They have suggested the advantage of this system is space and frequency diversity which is helpful in improving the performance of system. They have investigated space-frequency (SF) block coding performance for MIMO-OFDM along with different equalizers. They have suggested the optimum equalization method for the BER analysis.

In 2011, Jie et al. [24] suggested the combination of MIMO-OFDM system for the high data transmission rate. They have suggested that the combination in the condition of multi-path effects and frequency selective fading. They have found that the MIMOOFDM with STBC is better in BER performance in comparison to the absence of STBC.

In 2012, Darsena et al. [25] deals with MIMO broadband wireless communication systems, with the advent of employing OFDM with cyclic prefix (CP). It is used in the combination of STFBC. They have proposed two MIMO channel shortens. It is relying on linearly-constrained minimization of the meanoutput energy of the signal at the output of the channel shortened. Their results show that the without a substantial increase in computational complexity, with the proposed blind channel shortens method has a negligible performance penalty with respect to non-blind channel shorteners.
In 2012, Xin et al. [26] presented the analysis of Rayleigh fading channels based on discrete-rate adaptive modulation (AM) for MIMO-OFDM systems. For this the fading gain value is partitioned for each sub channel into a number of regions. It is adapted based on the modulation. They have obtained the spectral efficiency (SE) and average BER. Their results proved that the improved switching based $\mathrm{SE}$ is better than the traditional method.

In 2013, Seyman et al. [27] proposed a feed-forward multilayered perceptron (MLP) neural network trained with the Levenberg-Marquardt algorithm to estimate channel parameters in MIMO-OFDM systems. Bit error rate (BER) and mean square error (MSE) performances of least square (LS) and least mean square error (LMS) algorithms have been compared to their proposed method for performance evaluation and their result suggest that the neural network channel estimator has perform better than the LS and LMS algorithms

In 2013, Vakilian et al. [28] proposed a spacefrequency (SF) block coding scheme for MIMOOFDM system using antennas. The patterns used are reconfigurable radiation. They have assumed that the reconfiguration is done automatically. Their proposed scheme has the capability of spatial, frequency, and reconfigurable radiation pattern state diversity over frequency-selective fading channels. Their results suggest that their technique has the higher diversity and coding gain as to other SF codes.

In 2014, Falahati et al. [29] proposed orthogonal space-time-frequency block codes over frequencyselective with $\mathrm{m}$-coefficient Rayleigh fading channel bearing $2 \times 1$ and $2 \times 2$ MIMO-OFDM systems to compute the error rate performances.

In 2014, Doi et al. [30] presented a low complexity joint decoding scheme of block coded signals in an overloaded MIMO-OFDM system. They have proposed a two-step joint decoding scheme for the block coded signals. Their method is efficient in calculating metrics to reduce the number of the candidate codewords. It is because of the use of decoding based on joint maximum likehood symbol detection. Their proposed scheme is also efficient in reducing candidate codewords. They have shown that it reduces the complexity by about $1 / 174$ for 4 signal stream transmission.

In 2015, Shrivastava et al. [31] suggested the concept of multiple antennas for achieving good quality of 
service and high data rate communication in wireless system. They have suggested space-time-frequency (STF) codes for the efficiency in improving diversity gain. They have proposed the combination of beamforming with STF code got the performance improvement in terms of BER. They observed that the results are better than the STBC with beamforming.

In 2016, Chern et al. [32] suggested a MIMO-OFDM system that solves the problem of correlation between the transmitting and receiving antennas. They have suggested the combination of STBC with MIMOOFDM system. This system not considered the CP. They have verified the effects of mutual coupling based on BER.

In 2016, Chen et al. [33] presented a MIMO-OFDM system for the data hiding principle in the error correction coding channel. They have presented the impact of the antenna diversity, the maximum multipath delay and the Doppler shift. Based on this BER performance of the MIMO-OFDM has been presented. The accuracy of the MIMO-OFDM system has been outperformed in comparison to the SISO, OFDM and MIMO coding channel. The parameters considered are the BER of the carrier data, secret data and the data hiding capacity.

In 2016, Sharma et al. [34] suggested that the MIMO system has the capability of transmitting and receiving information from multiple antennas simultaneously and also efficient in the probability of information detection. They have integrated OFDM and MIMO system to improve spectral efficiency in conjunction with ISI reduction.

In 2017, Sezer et al. [35] proposed the optimal channel switching problem for average capacity maximization of the communication between the transmitter and also proposed the secondary receiver for the minimum average capacity fulfilment of the primary receiver and considering the average and peak power constraints. An alternative equivalent optimization problem has been provided by the authors for the solution of the optimization problem satisfies the constraints with equality. They have provided numerical examples to verify the theoretical investigations.

In 2017, $\mathrm{Li}$ et al. [36] discussed the $\mathrm{k}$-user MIMO interference channel with $M$ antennas at each transmitter and $N$ antennas at the corresponding receiver. They have proposed interference counteraction scheme to improve the global channel state information (CSI) for the receivers. Their proposed scheme performs better in terms of resisting the correlation of transmitters and accommodating interferences, if only the constraint on degrees of freedom provided.

In 2017, EI et al. [37] proposed a realistic and global simulation for predicting the behavior of a Radio over Fiber (RoF) system. They have considered a $2 \times$ 2 MIMO-OFDM RoF system at $60 \mathrm{GHz}$. It is based on spatial diversity (SD). It helps in increasing the reliability and spatial multiplexing (SMX) which increases data rate, but not necessarily reliability. They have evaluated and compared the performance of this system according to the various techniques like the diversity technique, modulation schemes, and channel coding rate for line-of-sight (LOS) desktop environment. They have found that $2 \times 2$ MIMOOFDM SMX system achieves a higher data.

In 2017, Namitha et al. [38] suggested the issue of MIMO-OFDM technique is its high peak-to-average power ratio (PAPR) over distinct antennas. Authors have been suggested that the selective mapping (SLM) may be helpful in reducing the PAPR without inducing any signal deformation in OFDM or MIMO-OFDM. The drawback of the SLM technique according to them is the need for sending side information (SI) with each OFDM data symbol for retrieving the transmitted data. They have presented a low complexity SLM technique using Hadamard sequence. They suggested that it substantially reduce the PAPR in MIMO-OFDM systems without the need for transmitting SI.

In 2017, Qiao et al. [39] suggested the hybridization of MIMO and OFDM for underwater acoustic (UWA) as it amplifies the required bandwidth efficiency. They have surveyed and presented the analysis in the direction of UWA MIMO-OFDM communication. They have compared the previous works based on the complexity and performance of the algorithms.

\section{Study analysis}

In this section an analysis has been presented based on the related reviews. Table 1 shows the method analysis table. This table describes the method, the approach used and the gaps based on the analysis of their method, results and future work suggested. This provides an overall view of the work along with the methodology impact and the contribution parameters used for the compariosn. 
Table 1 Method analysis

\begin{tabular}{|c|c|c|c|c|}
\hline S.No & Reference & Method & Approach & Problem statement \\
\hline 1 & {$[40]$} & $\begin{array}{l}\text { Semiblind } \\
\text { estimation }\end{array}$ & $\begin{array}{l}\text { The proposed method is immune to channel } \\
\text { zero locations, and is reasonably robust to } \\
\text { channel order overestimation. The proposed } \\
\text { method is applicable to the scenarios in which } \\
\text { the number of transmitters exceeds that of the } \\
\text { receivers. }\end{array}$ & $\begin{array}{l}\text { Other channel estimation } \\
\text { properties can be applied. }\end{array}$ \\
\hline 2 & [41] & $\begin{array}{l}\text { Particle swarm } \\
\text { optimization (PSO) and } \\
\text { genetic algorithm (GA) }\end{array}$ & $\begin{array}{l}\text { They have applied a combination of PSO and } \\
\text { GA for channel estimation in MIMO-OFDM } \\
\text { systems. Fine tuning has been enabled by LS } \\
\text { and MMSE channel estimation. The PSO and } \\
\text { GA technique has been considered to correlate } \\
\text { with the ideal model. }\end{array}$ & $\begin{array}{l}\text { Other evolutionay } \\
\text { techniques can be } \\
\text { validated for the better } \\
\text { results. }\end{array}$ \\
\hline 3 & {$[42]$} & Various system parameters & $\begin{array}{l}\text { They have analyzed the channel estimation in } \\
\text { MIMO-OFDM system. They have considered } \\
\text { the influence of various system parameters. }\end{array}$ & $\begin{array}{l}\text { It can be validated with } \\
\text { different transmitters and } \\
\text { receivers. }\end{array}$ \\
\hline 4 & [43] & $\begin{array}{l}\text { Performance Analysis in } \\
\text { MIMO-OFDM Systems }\end{array}$ & $\begin{array}{l}\text { They have studied several performance } \\
\text { analysis techniques in the case of MIMO- } \\
\text { OFDM system. }\end{array}$ & $\begin{array}{l}\text { Real time applications } \\
\text { based on the problem } \\
\text { satetment is missing. }\end{array}$ \\
\hline 5 & [44] & BER analysis & $\begin{array}{l}\text { They have applied data transmission with } \\
\text { OFDM-MIMO in the conjunction of AWGN } \\
\text { and Rayleigh fading channel. }\end{array}$ & $\begin{array}{l}\text { Different channel } \\
\text { capacity can be analyzed. }\end{array}$ \\
\hline 6 & {$[45]$} & $\begin{array}{l}\text { OFDM and multi-carrier } \\
\text { modulation } \\
(\mathrm{MCM})\end{array}$ & $\begin{array}{l}\text { They have presented turbo coded OFDM } \\
\text { systems and its channel estimation scheme for } \\
\text { reducing the PAPR in the system. }\end{array}$ & $\begin{array}{l}\text { Complexity related } \\
\text { parameter is missing. }\end{array}$ \\
\hline 7 & [46] & $\begin{array}{l}\text { BPSK Modulation Scheme } \\
\text { for MIMO-OFDM system }\end{array}$ & $\begin{array}{l}\text { BER using BPSK modulation technique and } \\
\text { optimum modulation have been analyzed. } \\
\text { Their working principle supports the better } \\
\text { noise reduction parameters. }\end{array}$ & $\begin{array}{l}\text { Carrer frequency offset } \\
\text { (CFO) can be analyzed. }\end{array}$ \\
\hline 8 & [47] & $\begin{array}{l}\text { Asynchronous multi-channel } \\
\text { media access control } \\
\text { protocol }\end{array}$ & $\begin{array}{l}\text { They have introduced media access control } \\
\text { protocols (MACs) for the lower layer of the } \\
\text { network. Their approach main functionality is } \\
\text { to provide access to a common media. }\end{array}$ & $\begin{array}{l}\text { The research replication } \\
\text { in different system is } \\
\text { missing. }\end{array}$ \\
\hline 9 & {$[48]$} & $\begin{array}{l}\text { Optimal thresholds for } \\
\text { discrete power levels using } \\
\text { adaptive modulation }\end{array}$ & $\begin{array}{l}\text { They suggested that the receiver mobility } \\
\text { allow the transmission thresholds and the } \\
\text { power level alternation determination which is } \\
\text { better in reduction in system's spectral } \\
\text { efficiency (SE). }\end{array}$ & $\begin{array}{l}\text { Multiuser proposition is } \\
\text { missing. }\end{array}$ \\
\hline 10 & [49] & 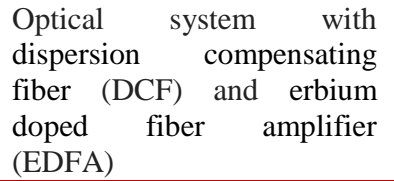 & $\begin{array}{l}\text { They have suggested DCF and EDFA for the } \\
\text { quality analysis with different error rate } \\
\text { parameters like BER, SNR, quality factor and } \\
\text { input power. }\end{array}$ & $\begin{array}{l}\text { The result impact can be } \\
\text { validated with different } \\
\text { parameters. }\end{array}$ \\
\hline
\end{tabular}

\section{Problem statement}

The major gaps identified by the analysis of the review work presented above are as follows:

1. The modulation scheme can be enhanced for the comparison and impact calculation with different parameter evaluation.

2. CFO can be analysed using BPSK modulation technique and optimum modulation.

3. Different channel capacity can be analyzed data transmission with OFDM-MIMO in the conjunction of AWGN and Rayleigh fading channel.
4. The channel estimation in MIMO-OFDM system can be considered in the influence of various system parameters.

5. Need of improvement in bit error rates with different channel capacity and channel fading variants.

\section{Conclusion}

In this paper an analytical review has been presented on the parametric study for enhancing the performance of MIMO-OFDM system. This study explores the gaps and the related methodology for the enhancement in the performance. For this several 
studies has been presented along with the analysis. Problem statement has been highlighted based on the literature presented. In future the gaps can be explored to enhance the parametric capability for improving the performance of MIMO-OFDM system.

\section{Acknowledgment}

None.

\section{Conflicts of interest}

The authors have no conflicts of interest to declare.

\section{References}

[1] Frederiksen FB, Prasad R. An overview of OFDM and related techniques towards development of future wireless multimedia communications. In radio and wireless conference 2002 (pp. 19-22). IEEE.

[2] Bingham JA. Multicarrier modulation for data transmission: an idea whose time has come. IEEE Communications Magazine. 1990; 28(5):5-14.

[3] Bhardwaj M, Gangwar A, Soni D. A review on OFDM: concept, scope \& its applications. IOSR Journal of Mechanical and Civil Engineering. 2012; 1(1):7-11.

[4] Hwang T, Yang C, Wu G, Li S, Li GY. OFDM and its wireless applications: a survey. IEEE Transactions on Vehicular Technology. 2009; 58(4):1673-94.

[5] Xia P, Giannakis GB. Design and analysis of transmitbeamforming based on limited-rate feedback. IEEE Transactions on Signal Processing. 2006; 54(5):185363

[6] Gesbert D, Shafi M, Shiu DS, Smith PJ, Naguib A. From theory to practice: an overview of MIMO spacetime coded wireless systems. IEEE Journal on Selected Areas in Communications. 2003; 21(3):281302.

[7] Paulraj AJ, Gore DA, Nabar RU, Bolcskei H. An overview of MIMO communications-a key to gigabit wireless. Proceedings of the IEEE. 2004; 92(2):198218.

[8] Gupta MV, Tiwari MR. Performance analysis and simulation result of MC-CDMA for AWGN channel and raleigh based on SNR/BER. International Journal of Advanced Computer Research. 2011; 1(1):13-7.

[9] Rathore M, Tanwar P. Reduction of co-channel interference in transmit/receive diversity (TRD) in MIMO System. International Journal of Advanced Computer Research. 2012; 2(5):13-7.

[10] Gupta P, Singh UP, Richhariya V. Analysis and comparison of the 4-PSK and 8-PSK STTC over Rayleigh fading channels for determining performance. International Journal of Advanced Computer Research. 2012; 2(5):140-6.

[11] Zhou S, Wang Z, Giannakis GB. Quantifying the power loss when transmit beamforming relies on finite-rate feedback. IEEE Transactions on Wireless Communications. 2005; 4(4):1948-57.
[12] Tarokh V, Seshadri N, Calderbank AR. Space-time codes for high data rate wireless communication: performance criterion and code construction. IEEE Transactions on Information Theory. 1998; 44(2):74465.

[13] Foschini GJ. Layered space-time architecture for wireless communication in a fading environment when using multi-element antennas. Bell Labs Technical Journal. 1996; 1(2):41-59.

[14] Anitha K, Sujatha BK. FPGA implementation of high throughput digital QPSK modulator using verilog HDL. International Journal of Advanced Computer Research. 2014; 4(14):217-22.

[15] Telatar E. Capacity of multi-antenna Gaussian channels. Transactions on Emerging Telecommunications Technologies. 1999; 10(6):58595.

[16] Daksh JK, Mohan R, Sharma S. Performance analysis with space-time coding in MIMO-OFDM systems with multiple antennas. International Journal of Advanced Computer Research. 2013; 3(10):126-9.

[17] Choubey R, Mohan R, Sharma S. A survey of BER performance of generalized MC DS-CDMA system. International Journal of Advanced Computer Research. 2013; 3(10):130-3.

[18] Hakobyan G, Girma M, Li X, Tammireddy N, Yang B. Repeated symbols OFDM-MIMO radar at $24 \mathrm{GHz}$. In European radar conference 2016 (pp. 249-52). IEEE

[19] Dubey AK, Khandagre Y, Kushwaha GR, Hemnani K, Tiwari R, Shrivastava N. PAPR reduction in OFDM by using modernize SLM technique. Recent trends in wireless and mobile networks. 2011(pp. 397-405.) Springer, Berlin, Heidelberg

[20] Hui CJ, Khatun S, Ali BM. Design of cross layer optimization of space-time block coded MIMOOFDM system. In conference on research and development 2006 (pp. 16-21). IEEE.

[21] Liu X, Bialkowski M. Investigations into the effect of spatial correlation on the performance of spacefrequency block coded MIMO-OFDM system. In antennas and propagation society international symposium 2007 (pp. 2439-42). IEEE.

[22] Yu X, Qiu R. Orthogonal space-time block codes in MIMO-OFDM cognitive radio communication system. IET international communication conference on wireless mobile and computing 2011 (pp. 107-11). IEEE.

[23] Gupta B, Saini DS. BER analysis of space-frequency block coded MIMO-OFDM systems using different equalizers in quasi-static mobile radio channel. In international conference on communication systems and network technologies 2011 (pp. 520-4). IEEE.

[24] Jie Z, Liang LI, Jin LI. Performance analysis of space time block code in MIMO-OFDM systems. In international conference on communication software and networks 2011 (pp. 13-6). IEEE.

[25] Darsena D, Gelli G, Paura L, Verde F. Blind channel shortening for space-time-frequency block coded 
MIMO-OFDM systems. IEEE Transactions on Wireless Communications. 2012; 11(3):1022-33.

[26] Xin Y, Yu X, Liu X, Chen X, Zhou T, Yin X. Discrete-rate adaptive modulation for MIMO-OFDM systems with space-frequency block code in Rayleigh fading channels. In spring congress on engineering and technology 2012 (pp. 1-4). IEEE.

[27] Seyman MN, Taşplnar N. Channel estimation based on neural network in space time block coded MIMOOFDM system. Digital Signal Processing. 2013; 23(1):275-80.

[28] Vakilian V, Frigon JF, Roy S. Space-frequency block code for MIMO-OFDM communication systems with reconfigurable antennas. In global communications conference 2013 (pp. 4221-5). IEEE.

[29] Falahati A, Payami M. Performance of orthogonal space-time-frequency block codes over highly frequency selective fading channel for $2 \times 1$ and $2 \times 2$ MIMO-OFDM systems. In Iranian conference on electrical engineering 2014 (pp. 1662-7). IEEE.

[30] Doi Y, Inamori M, Sanada Y. Complexity reduction in joint decoding of block coded signals in overloaded MIMO-OFDM system. IEICE Transactions on Communications. 2014; 97(4):905-14.

[31] Shrivastava N, Trivedi A. Combined beamforming with space-time-frequency coding for MIMO-OFDM systems. AEU-International Journal of Electronics and Communications. 2015; 69(6):878-83.

[32] Chern SJ, Huang WJ, Liu HI. The effects of mutual coupling for the space-time block coded MIMOOFDM systems. In international symposium on intelligent signal processing and communication systems 2016 (pp. 1-6). IEEE.

[33] Chen L, Fan Z, Huang J. Data hiding capacity of spatial domain bit replacement steganography in an MIMO-OFDM coding channel. AEU-International Journal of Electronics and Communications. 2016; 70(9):1295-303.

[34] Sharma V, Kaur H. On BER evaluation of MIMOOFDM incorporated wireless system. OptikInternational Journal for Light and Electron Optics. 2016; 127(1):203-5.

[35] Sezer AD, Gezici S. Optimal channel switching in multiuser systems under average capacity constraints. Digital Signal Processing. 2017; 63:145-54.

[36] Li Z, Song L, Shi H. Approaching the capacity of kuser MIMO interference channel with interference counteraction scheme. Ad Hoc Networks. 2017; 58:286-91.

[37] El Yahyaoui M, El Moussati A, El Zein G. On the capacity of MIMO-OFDM based diversity and spatial multiplexing in radio-over-fiber system. Optics Communications. 2017; 402:252-9.

[38] Namitha AS, Sameer SM. A bandwidth efficient selective mapping technique for the PAPR reduction in spatial multiplexing MIMO-OFDM wireless communication system. Physical Communication. $2017 ; 25: 128-38$.
[39] Qiao G, Babar Z, Ma L, Liu S, Wu J. MIMO-OFDM underwater acoustic communication systems-a review. Physical Communication. 2017; 23:56-64.

[40] Chen YS, Song JH. Semiblind channel estimation for MIMO-OFDM systems. EURASIP Journal on Advances in Signal Processing. 2012; 212:1-10.

[41] Vidhya K, Shankar Kumar K. Channel estimation of MIMO-OFDM system using PSO and GA. Arabian Journal for Science \& Engineering (Springer Science \& Business Media BV). 2014; 39(5):4047-56.

[42] Ngebani IM, Chuma JM, Zibani I, Matlotse E, Tsamaase K. Joint channel and phase noise estimation in MIMO-OFDM systems. In IOP conference series: materials science and engineering 2017 (pp. 1-4). IOP Publishing.

[43] Daksh JK, Mohan R, Sharma S. A survey of performance analysis in MIMO-OFDM systems. International Journal of Advanced Computer Research. 2013; 3(10):91-4.

[44] Verma P, Khare A. BER analysis on different channel based on OFDM and MIMO system. International Journal of Advanced Technology and Engineering Exploration. 2016; 3(18):62-6.

[45] Rani SR, Anzija IR, Hameed LS. Conceptual study of OFDM-Coding, PAPR reduction, channel estimation. International Journal of Advanced Computer Research. 2014; 4(15):686-92.

[46] Trivedi S, Raeen MS, Pawar SS. BER analysis of MIMO-OFDM system using BPSK modulation scheme. International Journal of Advanced Computer Research (IJACR). 2012; 2(5):219-26.

[47] Irandegani M, Bagherizadeh $M$. Designing an asynchronous multi-channel media access control protocol based on service quality for wireless sensor networks. International Journal of Advanced Computer Research. 2017; 7(32):190-9.

[48] Zaeem RM, Naeimi M, Arasteh ST, Chihi H. Optimal thresholds for discrete power levels using adaptive modulation in presence of imperfect channel state information. International Journal of Advanced Computer Research. 2017; 7(31):147-53.

[49] Abdulwahed SH, Abass TM. 8× 10 Gbps optical system with DCF and EDFA for different channel spacing. International Journal of Advanced Computer Research. 2016; 6(24):100-6.

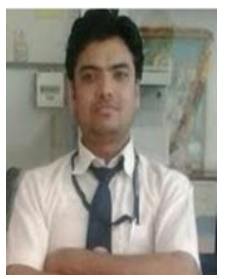

Rishi Choubey had completed his BE from SRIT, Datia, MP in 2007 in the Department of Electronics and Instrumentation. He completed his M.Tech in VLSI from Shri Ram Institute of Technology, Jabalpur, MP. Currently he is pursuing $\mathrm{PhD}$ in the department of Electronics and Communication from Swami Vivekanand University, Sagar, MP. His research areas are OFDM, MIMO and Channel Estimation.

Email: rishichoubey2012@gmail.com 